\title{
Traduire
}

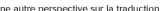

Revue française de la traduction

$225 \mid 2011$

Traduire hors des sentiers battus

\section{Traduire les expressions grossières en chinois}

Réflexion à partir de la traduction en chinois de « Monologue » de Simone de Beauvoir

\section{Florence Xiangyun Zhang}

\section{(2) OpenEdition}

1 Journals

Édition électronique

URL : http://journals.openedition.org/traduire/97

DOI : $10.4000 /$ traduire. 97

ISSN : 2272-9992

Éditeur

Société française des traducteurs

Édition imprimée

Date de publication : 15 décembre 2011

Pagination : 17-32

ISBN : 039-773X

ISSN : 0395-773X

Référence électronique

Florence Xiangyun Zhang, "Traduire les expressions grossières en chinois », Traduire [En ligne], 225 |

2011, mis en ligne le 10 février 2014, consulté le 10 décembre 2020. URL : http://

journals.openedition.org/traduire/97 ; DOI : https://doi.org/10.4000/traduire.97 


\section{Traduire les expressions grossières en chinois : \\ Réflexion à partir de la traduction en chinois de "Monologue " de Simone de Beauvoir}

\section{Florence Xiangyun Zhang}

Un soir de réveillon, tandis que partout les gens font la fête, Murielle, une femme abandonnée de tous - sa fille suicidée, son petit garçon à la garde du père, sa mère qui la juge, ses hommes envolés et le monde qui la condamne - se laisse aller, dans son désespoir, à son délire intérieur. Voici ce que met en scène Simone de Beauvoir au travers des propos violents, incohérents et ponctués de grossièreté de l'héroïne, dans "Monologue ", la deuxième nouvelle du recueil La Femme rompue, paru en 1967(1).

Dans le cadre du projet de publication d'une collection d'œuvres de Simone de Beauvoir(2), nous avons traduit cette nouvelle en chinois. Bien que l'auteur n'ait pas destiné ce " monologue " à la scène, le texte est profondément théâtral, et se prête facilement à une interprétation virtuelle chez le lecteur. Malgré le peu de ponctuation, les phrases sont simples, le style est oral et les gros mots nombreux. Traduire ce texte, c'est montrer ce personnage peu aimable, qui déteste tout le monde, et surtout restituer son discours injurieux, vulgaire, hystérique et fou. Dans cette entreprise, le défi majeur auquel nous nous sommes confrontés consiste en la traduction de l'expression grossière injurieuse(3).

Mais avant tout, faut-il traduire les gros mots ? L'héroïne de Beauvoir, proche de la folie, hait le monde entier et croit subir l'agression des hommes et des femmes complices, ainsi les mots qui la dominent ne sont autres que " con ", " salaud ", " chierie " ou " merdier ", et bien d'autres injures. Son monologue en chinois ne peut en aucun cas devenir un discours élégant et doit rester haineux et grossier. Nous nous devons donc de traduire ces gros mots.

(1) Simone de Beauvoir, 1967, La Femme rompue, Paris, Gallimard. Trois nouvelles sont regroupées dans ce livre : "L'Âge de discrétion ", "Monologue ", et "La Femme rompue ".

(2) À paraître aux éditions de Shanghai translations publications, Shanghai, 2011.

(3) Dans la nouvelle de Beauvoir, on rencontre également un grand nombre d'expressions argotiques dont la traduction nécessite d'autres réflexions. Dans le présent article, nous ne prêtons attention qu'à la traduction de gros mots qui ont un sens injurieux. Mais il nous ensemble en effet difficile de distinguer certaines expressions argotiques des gros mots. Même les ouvrages spécialisés avouent leur usage similaire. II convient alors d'y inclure le lexique injurieux de la langue parlée populaire. Voir Les Gros mots, P. Guiraud 1975 ; C. Rouayrenc, 1996. 
C'est pourtant délicat de mettre à l'écrit les gros mots, surtout en chinois, langue qui, jusqu'au début du xxe siècle, imposait des règles grammaticales et des vocabulaires distincts à la langue écrite(4) et à la langue parlée, à tel point que les expressions orales courantes n'étaient pas ou très peu écrites. Bien que le chinois moderne ait remplacé le chinois classique depuis un siècle, nous constatons encore un écart remarquable entre le parler et l'écrit(5). Entre autres, une bonne partie d'expressions vulgaires relèvent plutôt des langues parlées locales et il n'est pas toujours possible de les transcrire en écriture chinoise(6). Pour cette raison, les dictionnaires de langue chinoise ne répertorient qu'un petit nombre d'expressions populaires et orales du mandarin. Peu de linguistes chinois se lancent dans des recherches sur le gros mot(7), et parmi les rares études qui existent, nous remarquons que le corpus est majoritairement tiré d'œuvres littéraires.

Comme le dit justement Catherine Rouayrenc dans Les Gros mots(8) : "Le gros mot existe incontestablement et c'est un phénomène foncièrement oral ". Mais ce phénomène oral n'est pas uniquement linguistique. Puisque le gros mot heurte la bienséance, et que les tabous d'une société se manifestent à travers son emploi, ce phénomène est donc fondamentalement social, psychologique et même anthropologique(9). Ainsi la nouvelle de Beauvoir se prête-t-elle à de multiples lectures spécialisées. Faute de compétences dans ces divers domaines, et étant donné notre but de traduction, nous nous bornerons à nous poser les questions : Que veulent dire les gros mots? Est-il possible de traduire les gros mots ? Tous les gros mots français ontils un équivalent en chinois ? Quelle approche adopter pour les traduire?

(4) Le chinois classique ou le chinois littéraire (wenyan 文言) était la seule langue écrite pendant presque deux mille ans et ne connut que peu d'évolution. La réforme linguistique du début du xxe siècle a fait abandonner l'usage du chinois classique et institué le chinois écrit moderne proche du chinois mandarin oral.

(5) Ce n'est qu'en 1968 que le linguiste sino-américain Chao Yuan Ren (赵元任 1892-1982) déclare pour la première fois avoir travaillé spécifiquement sur un corpus de chinois oral pour établir sa "Grammaire du chinois parlé ". Voir A Grammar of Spoken Chinese (Berkeley, University of California Press, 1968), traduit en chinois en 1979 par Lü Shuxiang, Beijing, éditions Commercial Press.

(6) II est ainsi courant de voir des " $X$ " ou de petits carrés à la place de gros mots, que mettent des auteurs dans leurs écrits littéraires. Néanmoins, on remarque des écrivains comme Wang Shuo (王朔 1958-) qui créent des personnages populaires de Pékin chez lesquels les gros mots ne manquent pas. Les créations artistiques à caractère comique ont davantage tendance à utiliser le parler populaire, mais visant une diffusion nationale, elles sont en général marquées par les expressions et l'accent de la capitale. Nous constatons cependant que depuis quelques années, d'autres couleurs locales commencent à s'affirmer dans les fictions télévisées ainsi que dans d'autres représentations artistiques télévisuelles.

(7) Les études sur le gros mot sont souvent incorporées dans les études des injures. II existe en France, à part les ouvrages que nous citons ici, quelques dictionnaires qui répertorient les gros mots et injures. En Chine, les réflexions sont plus isolées ; et à notre grande déception, le corpus des études de linguistes chinois est constitué majoritairement, voire exclusivement, de dialogues de romans ou de pièces de théâtre connus, et manque de paroles spontanées enregistrées dans la vie réelle.

(8) C. Rouayrenc, Les Gros mots, collection "Que sais-je ", Paris, PUF, 1996, p. 6.

(9) Voir P. Guiraud, Les Gros mots, Paris, PUF, 1975, p. 8. 


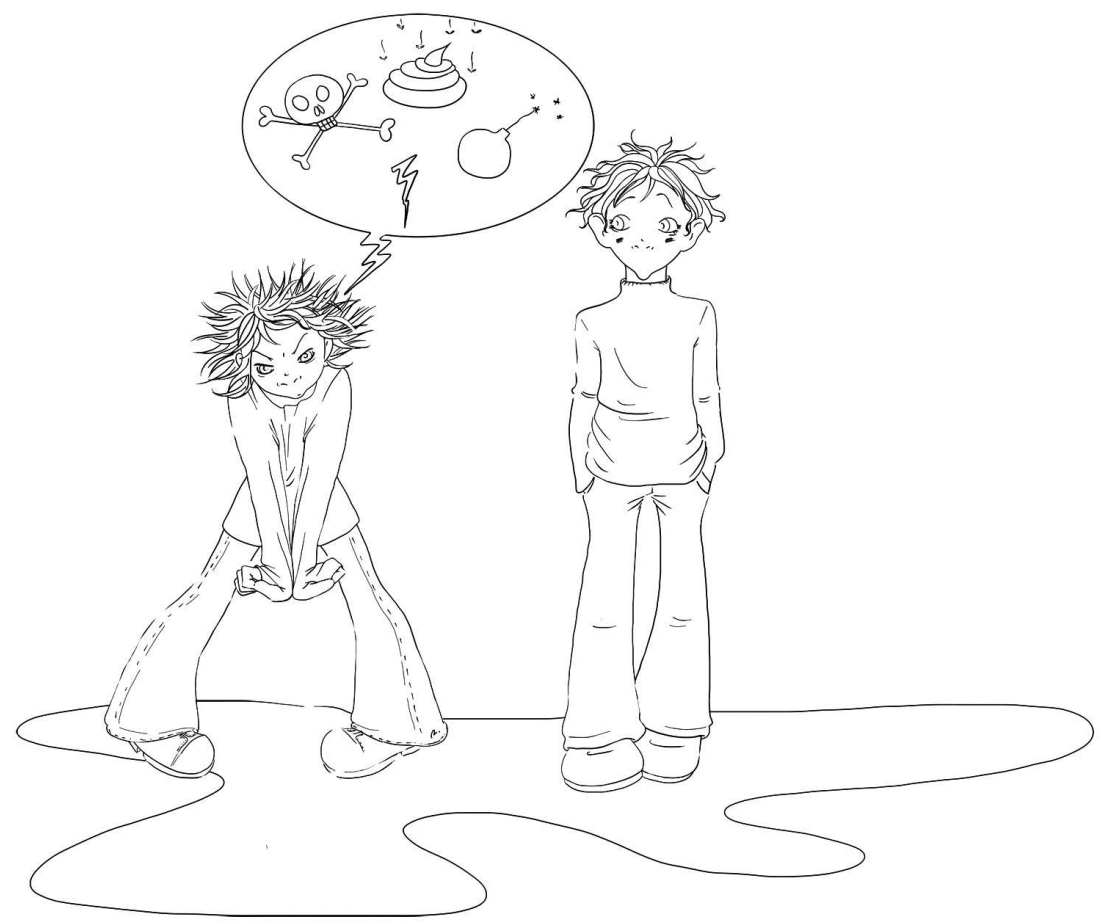

Illustration : Marlène Junius, http://alotoftralala.over-blog.com

En nous basant sur l'étude de Guiraud et de Rouayrenc, nous présenterons un échantillon de gros mots rencontrés dans le texte de Beauvoir, et évaluerons leur aspect notionnel et leur sens, c'est-à-dire leur emploi figuré dans le monologue ; nous analyserons par la suite l'utilisation de gros mots en chinois, et enfin les problématiques de la traduction en chinois de gros mots français.

\section{Gros mots et leur usage dans "Monologue "}

Sur la page du titre, Simone de Beauvoir cite Flaubert : "Elle se venge par le monologue ». Pour définir le terme monologue, Pierre Larthomas précise que le rôle du monologue est de faire " connaître les pensées, les intentions, les sentiments d'un personnage que nous ne connaîtrions autrement que par ses actes, sa mimique, et ses paroles "(10). De son côté, l'injure est " un acte de parole par lequel le locuteur affronte physiquement son adversaire ", " de la même

(10) Larthomas, Le Langage dramatique, Paris, PUF, 1980, 2001, p. 372. 
nature qu'un crachat symbolique „(11). Ainsi par ce monologue injurieux, Murielle ne racontet-elle pas seulement son histoire, ses sentiments et ses intentions, c'est aussi le moyen pour elle de " se donner raison ", de "récuser le jugement d'autrui "(12).

Le discours a lieu le soir du réveillon du Nouvel An. Murielle est toute seule dans son appartement alors que tout le monde fait la fête. Face aux bruits de la rue et de son immeuble, elle commence par dire " les cons! " et " salauds ils me déchirent les tympans "; quand elle parle de son frère et de sa mère, elle les appelle " chiards "; sa mère, dans son souvenir, "se baladait à travers son bordel de chambre à moitié à poil ". "Quelle chierie leurs fêtes ", " je n'en ai rien à foutre ". Elle se plaint de sa femme de ménage, "salope de Mariette elle me laisse en panne "; elle programme sa négociation avec son mari, dont elle est séparée, pour qu'il revienne, car " toutes ces pouffiasses elles ont un homme pour les protéger " ; elle se souvient de sa fille qui s'est suicidée quelques années auparavant, " je ne voulais pas qu'elle devienne une putain comme ma mère "; elle déteste son amie Dédé, " elle s'emmerde alors elle ramène son gros cul "; elle appréhende le lendemain où elle prévoit la négociation avec le mari, "ma nuit est foutue [...] demain ça foirera encore".

Dans cette panoplie de gros mots, beaucoup évoquent le domaine de la sexualité (" con", " bordel ", " foutre ", " putain ", " pouffiasse "), tandis que d'autres se réfèrent à l'excrément ( " chierie ", " foirer ", " s'emmerder ") ou à la puanteur (" salaud ", " salope ", " ordure ")(13). Évidemment, certains de ces mots semblent avoir une fonction référentielle(14). Dans la phrase "Je ne voulais pas qu'elle devienne une putain comme ma mère ", nous trouvons bien la signification propre du mot " putain ". Quelques mots qui désignent l'homosexuel conservent également leur signification. Par exemple, Murielle s'inquiète pour son fils en disant : " un gosse privé de sa mère finit toujours par mal tourner il deviendra un voyou ou une tante... ".

Mais quand on prononce "les cons ", s'agit-il vraiment de l'" organe sexuel de la femme " ? Évidemment non. Selon Guiraud, le " con " c'est l'" imbécile ", " celui qui ne comprend pas ", " sur lequel s'étend un mépris total "(15). II n'y a donc pas de métaphore sexuelle, et le mot n'implique pas l'image de la chose qu'il désigne. "S'emmerder " est employé juste au sens de " s'ennuyer ", ne faisant pas d'allusion à l'objet excrément. De même, " salaud " ou " salope " ne signifie pas une personne physiquement sale, mais exprime des sentiments de dégoût et d'hostilité.

(11) Guiraud, op. cit., p. 35.

(12) Francis et Gontier, Les Écrits de Simone de Beauvoir, la vie - l'écriture, Gallimard, 1979, p. 231.

(13) Voir Guiraud, op., cit.

(14) C. Rouayrenc, op. cit. Selon elle, nous distinguons trois fonctions du gros mot : fonction expressive, fonction référentielle et fonction démarcative. Quand la fonction expressive prime, la référence d'origine est effacée ; la fonction référentielle du gros mot désigne l'importance du signifié dans son emploi ; si le gros mot ressemble à un signe de ponctuation, il s'agit de sa fonction démarcative.

(15) Guiraud, op. cit, p. 65. 
C'est dire que dans la plupart des cas, les gros mots ne représentent plus dans l'esprit de ceux qui les emploient l'image de leur sens primitif. Comme le dit Guiraud(16), "les gros mots ont pour fonction de dévaloriser les choses qu'ils nomment en même temps que les gens auxquels ces choses s'appliquent ", et " cette dévalorisation implique une attitude affective de la part du locuteur ". C'est donc leur fonction expressive qui l'emporte sur le reste : ils ont un emploi figuré et sont utilisés quotidiennement pour traduire des sentiments fondamentaux le dégoût et la haine, le mépris et la dérision, ou l'ennui et le mécontentement(17).

\section{Les gros mots en chinois}

Si nous avons insisté sur la place marginale des gros mots dans le système écrit du chinois ainsi que dans les espaces publics en Chine, nous n'avons pourtant pas l'intention de nier l'existence réelle d'un grand nombre de gros mots(18). Dans sa "Brève histoire des injures en chinois", Liu Fugen énumère de nombreuses expressions grossières anciennes à connotation sexuelle.

En 1925, Lu Xun (1881-1936)(19) écrit un essai intitulé "Lun "Tamade" (论 "他妈的" À propos de Tamade) "(20) dans lequel il remarque l'omniprésence du gros mot :

Quiconque vit en Chine ne peut échapper à entendre souvent "tamade " ou des mots similaires. Je crois que cette expression est sans doute répandue partout où il y a des Chinois, et que la fréquence de son emploi n'est pas moins soutenue que les "bonjour " de politesse. Alors si on admet que la pivoine soit nommée "la fleur nationale " de la Chine, "tamade " devrait être "l'injure nationale " de la Chine(21).

(16) Ibid., p. 27.

(17) Ibid., p. 31.

(18) D'après "La brève histoire des insultes en chinois - étude du vocabulaire injurieux du chinois " (汉语詈词 研究--汉语骂詈小史, Liu Fugen, 2008), à partir du xl|e siècle (époque des Song), la littérature populaire, quoique marginalisée, nous laisse apercevoir la richesse du langage vulgaire.

(19) L'auteur d'une grande quantité de nouvelles, essais, contes, études littéraires et de traductions, Lu Xun est considéré comme le plus grand écrivain chinois du xxe siècle.

(20) L'expression tamade (他妈的) est l'euphémisme de cao tamade (操他妈的) en omettant le verbe grossier " cao ". L'expression signifie littéralement " baiser sa mère ", mais couramment employée, elle a perdu ce sens premier. Les variantes sont nombreuses : à la place de "sa mère ", cela peut être " ta mère ", " tes ancêtres ", " ta sœur ", etc. ; on peut également omettre le pronom possessif en disant : " made ".

(21) Voir "Lun Tamade ", La Tombe, OEuvres complètes de Lu Xun, Volume 1. 无论是谁 ，只要在中国过活 , 便总得常听到“他妈的”或其相类的口头禅。我想：这话的分布，大概就跟着中国人足迹之所至罢 ; 使 用的遍数，怕也未必比客气的“您好呀”会更少。假使依或人所说，牡丹是中国的“国花”，那么，这就 可以算是中国的“国骂”。”（论 “他妈的”，鲁迅全集第一卷）La traduction est de nous. 
Avec certaines variations comme made (妈的) ou nimade (你妈的(22)), tamade est effectivement le gros mot le plus utilisé des Chinois, non seulement à l'époque de Lu Xun, mais également aujourd'hui. Son étymologie montre la connotation sexuelle. Selon Lu Xun, dans un pays où priment la filiation et la famille, tamade reflète l'intention et même la stratégie des faibles d'humilier les ascendants de l'adversaire, pour ainsi faire davantage mal à celui-ci. À ses yeux, cette " injure nationale " bien que condamnable et méprisable, ne disparaîtra pas tant qu'il existe des puissants qui se vantent de leurs ancêtres. Sur ce point, Guiraud ne dit pas le contraire :

L'injure est l'expression d'une volonté de puissance. Mais d'une volonté de puissance inefficace et insatisfaite, qui n'est qu'un désir de puissance frustré et en fait, une impuissance. [...] impuissance du faible contre le fort, du petit contre le grand, de l'agresseur contre un ennemi hors d'atteinte, etc.(23)

Cependant, comme beaucoup de gros mots français, au fil du temps, tamade s'est banalisé et n'évoque plus, chez la plupart des utilisateurs et chez leurs interlocuteurs, le sens primitif extrêmement méchant. II est souvent utilisé comme juron, ou comme adverbe, ou encore inséré entre le sujet et le verbe pour marquer le ton grossier et hostile. Lu Xun prend note même d'un usage purement démarcatif d'une variante made :

"J'avais vu une fois, dans mon village natal, un paysan et son fils qui déjeunaient ensemble. Le fils montra un plat à son père en disant : ça c'est pas mal, "made" goûte un peu! Le père répondit : je n'en veux pas, "made" mange, toi ! On dirait (que ce mot) a presque le sens de "mon chéri" très en vogue aujourd'hui. „(24)

Ici le gros mot made est employé comme une ponctuation, et nous n'y trouvons en effet aucun sens péjoratif, mais le registre vulgaire est visible.

Il est clair qu'en français comme en chinois, le sujet de la sexualité occupe le terrain principal des gros mots. D'après Hu Shiyun(25), en chinois, les gros mots évoquant la sexualité sont perçus comme les plus malveillants. Ensuite viennent ceux ayant un lien avec la mort ou les maladies, et puis ceux qui font référence à des espèces inférieures (animaux). Un certain nombre d'insultes impliquent la filiation : le fait d'appeler son adversaire "mon petit-fils " ou de lui dire " je suis ton ancêtre " est insultant, et ces mots normaux deviennent gros mots dans ce

(22) Dans notre étude, s'agissant des mots clés en chinois de la démonstration, nous mettons la transcription phonétique en italique dans le texte, et les caractères chinois entre parenthèses quand ils paraissent pour la première fois.

(23) Guiraud, op. cit., p. 119.

(24) Lu Xun, op. cit.我曾在家乡看见乡农父子一同午饭, 儿子指一碗菜向他父亲说：《这不坏, 妈的你尝 尝看！》那父亲回答道：《我不要吃。妈的你吃去吧！》则简直已经醇化为现在时行的“我的亲爱 的”的意思了。 La traduction est de nous.

(25) Voir Hu Shiyun, 1997. 
genre de situation. En revanche, la référence à l'excrément et à l'ordure est relativement moins importante, néanmoins le mot pi (屁 pet) apparaît très souvent dans le langage familier pour dévaloriser l'adversaire.

En réalité, le sens du gros mot dépend des circonstances où il est utilisé, des personnes qui le prononcent et qui le reçoivent, et aussi de la façon dont il est prononcé. Hu Shiyun constate que les mêmes gros mots ne font pas réagir de la même manière selon la situation, l'intonation, et que dans certains cas, l'emploi de gros mots peut changer l'ambiance et la rendre plus conviviale ou intime(26).

Selon Rouayrenc, " est gros mot ce que les gens considèrent comme tel, c'est-à-dire tout ce qui relève d'un domaine tabou, et/ou passe pour injurieux "(27). Puisqu'il y a des tabous communs dans les cultures différentes et des tabous propres à chaque société, certains gros mots chinois peuvent correspondre à certains gros mots français, mais cela ne veut pas dire que chaque gros mot français trouve un équivalent en chinois.

\section{Problématique de la traduction du gros mot}

Nous avons vu que les gros mots et l'injure occupent une place essentielle dans la nouvelle de Beauvoir, mais les images qu'ils comportent ne sont que secondaires par rapport au sentiment qu'ils expriment.

Ainsi les expressions telles que "les cons " et " je n'en ai rien à foutre " sans cesse répétées par l'héroïne ne concernent-elles pas le sujet du sexe, mais dénoncent seulement le mépris. II nous semble donc évident, dans une approche de traduction, de ne pas nous intéresser à la signification propre de ces gros mots, mais de nous focaliser sur les émotions que ces expressions laissent entendre.

Or, comme nous l'avons mentionné, le gros mot est symbole d'une impuissance, et traduit l'expression naturelle d'un "réflexe de dévalorisation et de dépréciation „(28), il est donc clair que les autres mots ne peuvent remplir ce rôle. Comment établir les mêmes liens entre le mot et le sentiment? Nous avons suivi cinq démarches différentes:

\section{a. Conserver l'équivalent}

$\|$ y a des gros mots en chinois dont la signification et l'emploi correspondent à certains gros mots français, et nous n'avons qu'à en choisir selon le contexte.

(26) Hu Shiyun, op. cit.

(27) Rouayrenc, op. cit., p. 7.

(28) Guiraud, op. cit., p. 123. 
Ex.(29) 1) Toutes ces pouffiasses, elles ont un homme pour les protéger des gosses pour les servir et moi zéro. (p. 93)

这帮骚女人全都有男人保护有孩子伺候就我一无所有

Ex. 2) Je ne voulais pas que ma fille devienne une putain comme ma mère. (p. 95)

我可不想让我的女儿成了婊子象我妈一样

Dans ces deux phrases, "pouffiasse " et "putain " ont le sens de prostituée, le mot presque universel pour dévaloriser la femme. En chinois, il existe également plusieurs expressions similaires, il est donc aisé d'en choisir en fonction de la situation. "Pouffiasse " du premier exemple désigne des femmes que la locutrice voit dans son immeuble, et qui sont des personnes non définies, tandis que " putain " évoque sa mère qu'elle hait. Nous pensons juste, pour traduire " putain ", de mettre biaozi(30) (婊子) dont le sens est plus fort que saonüren (骚女人) pour " pouffiasse".

Ex. 3) Salaud de Tristan je veux qu'il m'invite au restaurant au théâtre [...] tout ce qu'il sait faire c'est de s'amener ici seul [...] Même cette nuit pas un geste ! Salaud! (p. 96)

特里斯丹这个混蛋我要他带我去餐厅去剧场 [...]他就知道一个人过来 [...] 连今天晚上都没个表示! 王八蛋!

Ex. 4) Un million d'enfants massacrés et après ? Les enfants ce n'est jamais que de la graine de salauds, ça désencombre un peu la planète, ils reconnaissent qu'elle est surpeuplée alors quoi? (p. 103)

有一百万儿童被杀害后来呢? 儿童还不就是没长大的恶棍杀掉了地球就 松快一点谁都知道世界上人太多了不是吗?

Dans l'exemple 3, le mot " salaud " est utilisé deux fois pour désigner Tristan, le mari séparé de l'héroïne, que celle-ci tente de récupérer ; dans l'exemple 4, " salaud " désigne les enfants dans le monde. Si, à l'origine le mot fait référence à la saleté, l'usage lui donne plutôt le sens de la méchanceté qui inspire le dégoût ou la haine. Pour traduire le même mot, nous avons sélectionné hundan (混蛋 ), wangbadan (王八蛋) et egun (恶棍) qui peuvent rendre ce sens. Hundan et wangbadan nous semblent plus appropriés pour des personnes précises dans un face-à-face direct, et egun est plus adapté dans des situations plus vagues. Si l'usage du deuxième ressemble à celui du premier, nous trouvons que son sens est plus fort, ce qui convient au ton du discours : après avoir précisé le comportement de Tristan, la haine de Murielle ne peut qu'être plus forte.

(29) Tous les exemples sont tirés du "Monologue " (La Femme Rompue, 1967, Gallimard). Pour ces premiers exemples traduits, nous ne fournissons pas d'explication en français de la phrase chinoise. Car il ne s'agit pas de remaniement de phrase, mais seulement du choix lexical.

(30) Nous mettons en caractère chinois et en pinyin, le système chinois de transcription phonétique en lettres latines, les mots dont nous expliquons le choix. 
Ce genre d'équivalent n'est pourtant pas toujours facile à trouver sans imagination. Dans l'exemple suivant, le mot " momie " (31) demande un traitement plus libre.

Ex. 5) Cette momie ça donne le frisson d'imaginer son entre-jambes elle dégouline de parfums mais par en dessous elle sent, elle se maquillait elle se pomponnait elle ne se lavait pas ce que j'appelle se laver [...] (p.105)

这个老不死的她在大腿之间喷的香水都流出来了可还是有味儿她成天化 妆喷香水可她不洗澡反正我不认为她那是洗澡 [...]。

La " momie ", prenant l'image de la momie égyptienne, désigne dans le langage familier, selon le Larousse, une personne sèche, maigre et inactive. Cependant, dans cette phrase, Murielle parle de sa mère de soixante-dix ans qu'elle soupçonne d'être l'auteur de bien des complots contre elle. Elle pense même que sa mère est responsable de tous ses malheurs. La forme physique et l'inactivité ne sont pas caractéristiques de cette femme, mais il s'agit plutôt de sa vieillesse. Pour insulter une personne vieille en chinois, laobuside 老不死 的 (le vieux qui ne meurt pas) est l'expression la plus méchante, et correspond au ton haineux du personnage.

\section{b. Expliquer la signification}

Nous avons dit plus haut que beaucoup de gros mots français ont perdu leur sens primitif et ont été banalisés, tandis que d'autres gros mots conservent toujours leur référence première. C'est le cas des mots qui désignent l'homosexualité, la race ou l'origine ethnique.

Ex. 6) Nanard avec ses cinq enfants c'est tout de même une tantouze. (p. 89) 纳纳尽管有五个孩子也还是被人操的

Nanard est le frère de l'héroïne qu'elle n'aime pas non plus. Le mot " tantouze " désignant l'homosexuel passif n'a pas d'équivalence connue en chinois. Si nous mettions le mot neutre d' " homosexuel " - tongxinglian (同性恋), le sens péjoratif et la grossièreté seraient perdus. II serait absurde également de se tourner vers la motivation du mot « tantouze " et son lien avec le mot " tante ". Nous avons donc choisi d'utiliser le participe passif du verbe grossier "baiser "-cao (操) : " bei ren cao de (被人操的) : celui qui se fait baiser ", ce qui explicite le sens du mot et en même temps conserve entièrement la grossièreté. Par contre, quand il s'agit de gros mots relatifs au racisme, la solution est moins évidente :

Ex. 7) Je ne suis pas raciste mais je m'en branle des Bicots des Juifs des Nègres juste comme je m'en branle des Chinetoques des Russes des Amerlos des Français. (p. 102)

我没有什么种族歧视可我讨厌所有这些阿拉伯人犹太人黑鬼也一样讨厌 中国人俄国人美国佬和法国人。

(31) Le mot « momie " n'est pas un gros mot, bien entendu, s'il n'a pas de sens injurieux. Voir supra, note 27. 
Dans cette phrase, les mots à connotation raciste sont nombreux. Si nous trouvons des mots relativement péjoratifs pour traduire "Nègres " (heigui 黑鬼) et "Amerlos " (meiguolao 美国佬), les Chinois, éloignés de l'histoire coloniale occidentale, ne connaissent pas le racisme envers les Arabes. De ce fait, la traduction de "Bicot " pose problème, et bien entendu celle de "Chinetoques " qui signifient les Chinois mêmes. Compte tenu du sens négatif de l'ensemble de la phrase, nous avons pris la décision de les traduire par des mots neutres, ce qui fait perdre, évidemment, le racisme profond derrière l'existence de ces mots. Nous ne cherchons pas pour autant à mettre une note de bas de page pour fournir une explication supplémentaire, puisque le style hystérique et déraisonné du discours n'exigerait pas du lecteur une volonté d'érudition. En réalité, il est inévitable quelquefois de s'avouer que certains éléments culturels ne passent pas dans la traduction.

\section{c. Choisir un autre gros mot}

Si le gros mot n'a pas d'équivalent dans la langue d'arrivée, et que sa fonction référentielle n'est pas prise en compte, il n'y a donc pas de contenu sémantique à expliquer. C'est le cas des jurons. Le gros mot utilisé comme juron sous forme d'interjection sert en général à exprimer un sentiment spontané face à une situation donnée. Le sens premier du mot n'est pas pris en considération par le locuteur.

Ex. 8) Merde alors! Je veux qu'on me respecte je veux mon mari mon fils mon foyer comme tout le monde. (p. 94)

真他妈的! 我就是想让别人尊重我一点儿就是想有丈夫儿子还有一个家 跟其他人都一样。

Si en français, " merde " figure parmi les jurons les plus courants, il en va de même pour tamade 他妈的 en chinois, l'“ injure nationale " chinoise dont nous avons parlé plus haut, si bien que les apprenants sinophones le prennent systématiquement pour l'équivalent du mot français (32). Ici nous ajoutons l'adverbe zhen 真 devant tamade pour renforcer le ton et qui assure en même temps le lien avec la phrase précédente.

À part les jurons, nous rencontrons également certains verbes dont l'usage nous impose la même tactique :

Ex. 9) La garce elle me vitriole et il faut que je la boucle. (p.105)

这个臭女人她作践我还不让我说话 ${ }^{33}$ 。

(32) Alors que tamade ne peut traduire " merde " que lorsque celui-ci est sous forme de juron et que la situation le permet. Nous ne le considérons donc pas comme l'équivalent de " merde ", mais un autre mot.

(33) L'expression " la boucler " dans le sens de " se taire " n'a pas d'équivalent en chinois, nous avons donc choisi de la rendre avec des mots ordinaires : elle ne me laisse pas parler ( 不让我说话 ). Voir l'analyse suivante sur la renonciation au gros mot. 
Dans cette phrase, le mot " vitrioler " qui ne fait pas référence au vitriol demande une interprétation. Dans le texte, l'héroïne, condamnée par tous, rongée par la solitude le soir du réveillon, téléphone à sa mère pour tenter une réconciliation, mais celle-ci ne veut pas lui parler. Elle se sent offensée et haïe alors elle prononce ces mots, pourtant elle n'est pas défigurée par sa mère. II nous faut donc rendre le verbe par un mot familier mais surtout dur et cruel. Zuojian 作践 (piétiner, humilier) correspond bien à cette situation, même si sa signification n'est pas identique à celle de "vitrioler ".

Quelquefois, nous ne pouvons pas nous limiter à la même fonction grammaticale du mot, c'est dire qu'il n'est pas toujours possible de remplacer un gros mot en tant que nom par un autre nom.

Ex. 10) [...] ils se collent des boules Quiès mentales dans les oreilles et si une phrase se faufile à travers ils répondent des conneries (p. 100)

他们的耳朵全塞住了万一偶然听见一句话他们就回答得狗屁不通。

Ici, Murielle se plaint des autres qui ne l'écoutent pas parler. Le nom " conneries " correspondrait en chinois à huhua 胡话, shahua 俊话 (parole insensée), or ces mots ne faisant pas partie du registre vulgaire, ne pourraient montrer le ton désagréable ni le dégoût de Murielle envers son entourage. II est sans doute nécessaire de ne pas chercher un nom pour traduire un nom, mais viser le contenu du gros mot. C'est ainsi que nous pouvons penser à la locution verbale hushuobadao 胡说八道 (dire des choses insensées) ou à l'expression grossière adjectivale goupibutong 狗屁不通 (nul, illogique, insensé ; littéralement : le pet du chien qui n'est pas fluide). Par rapport à la première, la seconde expression est plus vulgaire et nous semble plus appropriée.

\section{d. Compenser au niveau de la phrase}

Les trois démarches précédentes traitent en effet de gros mots individuels, alors que nous rencontrons souvent des locutions grossières dont la traduction demande plus de réflexion.

Ex. 11) Toujours de l'argent ce que les hommes sont bas ils croient que tout peut s'acheter d'abord son fric j'en avais rien à foutre c'était des clopinettes à côté de ce que me verse Tristan. (p. 98)

又是钱这些男人都太小人还以为拿钱什么都能买其实我要他的钱有屁用 那一点钱也就是特里斯丹给我的零头。

Dans ce passage, Murielle se rappelle son premier mari Albert, le père de sa fille suicidée, qui, quelques années avant la mort de celle-ci, voulait reprendre la garde de leur fille en proposant de l'argent à Murielle. Murielle ne l'a pas laissé faire. L'expression " n'en avoir rien à foutre " est employée à plusieurs reprises dans le texte. Elle est effectivement parmi les expressions grossières les plus courantes en français qui possèdent bien des synonymes. Pourtant, en 
chinois, il nous paraît difficile de trouver un mot ou une locution équivalente. Notre regard se dirige alors vers la phrase. Dès lors que nous ne nous focalisons pas sur une expression particulière mais sur " son fric j'en avais rien à foutre c'était des clopinettes à côté de ce que me verse Tristan ", nous saurons qu'il y a des gros mots en chinois à notre disposition qui peuvent participer à l'expression du même sens et du même sentiment. Dans 《我要他的钱有屁用 (littéralement : son argent me sera utile comme un pet) ", c'est encore une fois le caractère pi 屁 (pet) qui définit le niveau du langage équivalent à l'original.

En fait, si ce genre d'expression nous perturbe souvent, c'est que, comme la plupart des traducteurs, nous n'arrivons pas facilement à nous détacher de l'image d'origine qu'elle évoque, d'autant plus qu'elle est inhabituelle dans notre travail. Or, nous le savons déjà, l'utilisation de beaucoup de gros mots exprime surtout le désir de choquer et de provoquer(34). En d'autres termes, le choix de gros mots peut être entièrement aléatoire chez une personne qui, emportée par la colère, prononce tout ce qui est désagréable pour son adversaire.

Devant la phrase suivante, nous savons donc considérer l'ensemble au lieu de fixer l'attention sur des mots et des locutions.

Ex. 12) Je peux bien clamecer avec mon pauvre cœur surmené personne n'en saura rien ça me fout la trouille. (p. 96)

我可怜的心脏这么超负荷我没准什么时候就完蛋了可连一个人都不知道 这太吓人了。(Littéralement en français : mon pauvre cœur tellement surmené je vais peutêtre crever un de ces jours mais pas une personne n'en saura rien c'est vraiment effrayant.)

Après avoir cherché en vain une traduction toute prête de "ça me fout la trouille ", nous pensons judicieux de ne pas prêter attention à la locution, mais d'employer un gros mot à un autre endroit de la phrase pour conserver le niveau du langage. Nous avons donc traduit cette expression avec des mots normaux, et utilisé wandan 完蛋 (être foutue, crever), il nous semble que le même sens est rendu. La présence d'un gros mot, selon nous, suffit pour montrer le ton désespéré du personnage.

Ex. 13) La télé aussi quelle bande de cons! J'étais faite pour une autre planète je me suis trompée de destination. (p. 107)

电视真他妈的无聊！我真该降生在另外一个星球上可惜投错了胎。

Ici avec "quelle bande de cons ", Murielle montre du doigt des personnes ou des émissions qui sont à la télévision. Or, si " con " dans le sens d'» imbécile " peut s'appliquer aussi bien à des personnes qu'à des choses, en chinois il nous paraît improbable d'employer sha (俊) ou

(34) Nous tenons ainsi à faire une remarque. L'anecdote de Zidane au match final de la Coupe du Monde de football en 2006 a été rapportée dans les journaux chinois. L'insulte adressée à Zidane par le joueur italien étant " fils de pute ", les journaux avaient traduit par " 你母亲是妓女 (ta mère est une prostituée) ". Le lecteur chinois imaginait bien que c'était insultant, mais trouvait la formule trop écrite et sérieuse pour être provocatrice. Pourtant, il existe bien des gros mots qui ont le même sens. 
chun (蚌) s'agissant de la télévision. Le choix s'est imposé sur wuliao (无聊, sans intérêt, ennuyeux) qui convient surtout à la télévision. Cependant puisque wuliao (无聊) n'est pas du registre vulgaire, nous ajoutons tamade ( 他妈的) entre le sujet et le verbe pour que le ton reste identique à celui de l'original.

De cette manière-là, nous ne traduisons pas telle ou telle expression, mais nous traduisons des phrases. Les phrases traduites contiennent, comme les phrases originales, des gros mots, et s'ils ne correspondent pas complètement à ceux de l'original, l'ensemble du sens et du sentiment est rendu.

\section{e. Renoncer au gros mot}

Dans certains cas, nous devons reconnaître qu'il faut parfois renoncer au gros mot et nous contenter du style parlé du texte traduit.

Ex. 14) Ils rigoleront jaune quand Tristan me reviendra. II reviendra je l'y forcerai bien. Je retournerai chez les couturiers je donnerai des soirées. [...] Ils seront salement baisés [...] (p. 97)

等特里斯丹回来看他们怎么笑。他肯定会回来我要逼他。我还会到时装 店去做衣服 $[. .$.$] 他们一定气得要死。$

Dans ce passage, l'héroïne s'adresse à tous ceux qui fêtent le Nouvel An dans son immeuble. Elle imagine qu'ils se moquent d'elle, et qu'elle se vengera quand son mari reviendra. " lls seront salement baisés ", c'est une victoire purement imaginaire ; et le mot "baisé " selon nous, ne signifie pas "dupé " comme l'indique le Larousse, mais amplifie le désir de l'héroïne d'énerver ou d'enrager les autres. C'est ainsi que nous traduisons par qi de yaosi (气得要死, énervé à en mourir). Bien que si (mourir) tout seul ne soit pas un gros mot, son usage dans le langage parlé représente toujours une intention d'exagérer les sentiments, ce qui correspond donc au texte original.

L'exemple suivant est aussi révélateur du problème de la traduction de certaines expressions. Nous avons eu recours, dans le chapitre précédent, à la compensation au niveau de la phrase par d'autres gros mots, ce qui ne peut pourtant être la solution systématique, en fonction de la syntaxe de chaque phrase.

Ex. 15) Ça me fait mal de me rappeler ce temps-là personne ne me sort plus je reste là à me faire chier. (p. 96)

想起那段时间真叫我难受后来就再也没人带我出去我就成天呆着犯俊 (littéralement : quand je me rappelle ce temps-là ça me fait mal après plus personne ne me sort je reste tout le temps plantée là comme une sotte)

Ex. 16) Moi ça me cassait le cul de jouer à la dame de fréquenter des emmerdeurs. (p. 102) 我特别讨厌玩阔太太那一套还得穷应酬 (littéralement : je déteste très fort jouer au tralala de la dame riche et aller sans intérêt dans les trucs mondains.) 
Dans ces exemples, les expressions " se faire chier " et " casser le cul ", comme celles que nous avons traitées plus haut, ne trouvent pas d'équivalent en chinois. Cependant l'organisation syntaxique de ces phrases ne nous incite pas à y insérer de gros mots tels que tamade. Nous choisissons alors de renoncer à employer de gros mots, et de veiller à ce que le texte garde le même style parlé et le registre populaire. Daizhe fansha ( 呆着犯俊, rester plantée là comme une sotte), façon de parler orale et populaire, montre bien l'état où se trouve Murielle ; nayitao (那一套, le tralala) ainsi que l'adverbe populaire qiong (穷, sans but, sans intérêt) reflètent l'ennui et le dégoût de l'héroïne face à la vie qu'elle menait avec Tristan.

À travers la traduction des derniers exemples, nous pensons qu'il est parfois possible de ne pas traduire le gros mot, et que les expressions populaires avec la syntaxe du style oral peuvent remplir le rôle du gros mot et produire le même effet.

\section{Conclusion}

Quand un texte littéraire commence par "les cons! " et que les pages suivantes sont remplies de gros mots, il n'est pas étonnant que le traducteur soit saisi et même obsédé par cette présence peu habituelle. Après quelques lectures, les gros mots semblent laisser place à l'image de la femme qui témoigne son désespoir avec un dégoût exagéré de la vie et un excès de haine envers le monde entier. En effet, ce dégoût et cette haine sont rendus plus explicites par la multiplication de gros mots.

D'après Guiraud (35), par le gros mot s'exprime une volonté de dévalorisation et de dépréciation. Puisqu'il représente à l'origine des sujets tabous, la force d'attaque et de provocation du gros mot est incomparable aux autres modes d'expression.

Dans une approche de traduction, nous ne devons donc pas faire abstraction de gros mots, d'autant plus qu'ils existent dans toutes les langues. Or s'il y a des tabous communs à différentes cultures, d'autres sont propres à chaque société, et l'image représentée par certains gros mots français n'évoque pas de sentiments particuliers dans une autre langue. Ainsi fautil non pas un traitement uniforme dans la traduction de gros mots, mais des tactiques variées adaptées à chaque situation.

Le cas le plus simple, c'est bien entendu quand on trouve dans la langue d'arrivée, en l'occurrence le chinois, les mots qui correspondent à l'image et à l'emploi de ceux de l'original. Nous pouvons les prendre comme équivalents. Quant à ceux qui renvoient à une référence méconnue en chinois, nous devons traduire le signifié ou l'expliquer, tout en conservant le même registre. Nous remarquons également que, dans l'usage quotidien, beaucoup de gros mots n'évoquent pas leur image tabou d'origine ; devenus banalisés, certains d'entre eux sont

(35) Guiraud, 1975. 
employés par un grand nombre de personnes pour exprimer des sentiments négatifs. C'est le cas des jurons. Pour les traduire, il nous semble souhaitable d'exploiter les ressources en la matière de la langue chinoise : choisir un juron chinois que les Chinois proféreraient dans la même situation.

D'autres emplois de gros mots sont plus complexes et nécessitent plus de créativité de la part du traducteur. Pourtant les gros mots " n'ont de sens que s'ils sont compris et utilisés par tout le monde " (36), et "pour qu'il y ait insulte, il faut absolument qu'elle soit perçue comme telle "(37). II nous faut être conscients qu'il n'est pas possible d'imaginer et d'inventer de gros mots en traduction. S'il n'y a pas d'expressions en chinois équivalentes à des locutions grossières françaises, nous devons prendre l'ensemble de la phrase en considération et ne pas hésiter à insérer un autre gros mot courant, lorsque la syntaxe le permet, pour compenser la perte de grossièreté. Parfois, il est aussi nécessaire de renoncer au gros mot si l'organisation de la phrase ne lui laisse pas d'espace.

En résumé, si l'emploi de gros mots fait partie du style de l'œuvre que le traducteur a l'obligation de rendre, tous les gros mots ne sont pourtant pas signés d'une intention particulière. Il convient alors de les mesurer cas par cas. Quand le choix du gros mot dans le texte original nous paraît aléatoire, nous pouvons nous donner la liberté d'en choisir un selon la situation, le sentiment exprimé, mais également en fonction de l'organisation syntaxique de notre texte. Le plus important, c'est de restituer, dans un texte extrêmement oral, le ton du désarroi et une note de grossièreté.

xyunzhang@yahoo.com

Florence Xiangyun ZHANG est maître de conférences à I'UFR Langues et Civilisations d'Asie orientale, Université Paris Diderot, où elle enseigne la langue chinoise et la traduction français-chinois. Elle a exercé le métier de traducteur-interprète durant plusieurs années avant de commencer une thèse de doctorat en traductologie qu'elle a soutenue en 2006, intitulée "Traduire le théâtre, l'application de la théorie interprétative à la traduction en chinois d'œuvres dramatiques françaises ", à I'ESIT, Université Paris Sorbonne Nouvelle.

Ses sujets de recherche : la traduction de textes dramatiques, la traduction de textes littéraires au style oral, la traductologie en Chine, et l'enseignement de la traduction à l'université. Elle continue de traduire de la littérature française pour des éditeurs chinois. Ses traductions récentes : La Consolante d'Anna Gavalda, La Femme rompue de Simone de Beauvoir, Au temps du Bœuf sur le toit de Maurice Sachs.

(36) Boumard, 1979, Les gros mots des enfants, p. 122.

(37) Rouayrenc, 1995, p. 112. 


\section{Bibliographie :}

BOUMARD Patrick, 1979, Les gros mots des enfants, Paris, Stock.

DE BEAUVOIR Simone, 1967, La Femme rompue, Paris, Gallimard.

FRANCIS Claude et GONTIER Fernande, 1979, Les Écrits de Simone de Beauvoir, la viel'écriture, Paris, Gallimard.

GUIRAUD Pierre, 1975, Les Gros mots, Paris, PUF, collection "Que sais-je ? ".

HU Shiyun, 1997, “À propos des insultes et d'études sur les insultes (骂人话及骂人话研究 杂谈) ", dans Enseignement et recherche linguistiques (Yuyan jiaoxue yu yanjiu 语言教学与 研究), http://www.cnki.com.cn/Journal/F-F1-YYJX-1997-03.htm, consulté le 12 mai 2011.

LARGUĖCHE É., 1983, L'effet injure, de la pragmatique à la psychanalyse, Paris, PUF.

LI Rulong, 2007, "Regard sur l'étude du lexique oral et du lexique écrit du chinois (关注汉 语口语词汇与书面语词汇的研究 ) ", dans Journal de l'Université normale de Shaanxi, n 3/2007, http://www.eyjx.com/1/ReadNews.asp?NewsID=4391, consulté le 08 mai 2011.

LIU Fugen, 2008, La brève histoire des insultes en chinois - étude du vocabulaire injurieux du chinois " (汉语䍗词研究一汉语骂䍗小史), Hangzhou (Chine), éditions Zhejiang Renmin Chubanshe.

LU Xun, 1981, "Lun "tamade" ", dans CEuvres complètes, Beijing, mise en ligne sur http://www.ziyexing.com/luxun/luxun_zw_fen_22.htm 